JOURNAL OF

SYMPLECTIC GEOMETRY

Volume 9, Number 3, 387-401, 2011

\title{
POLYNOMIAL POISSON STRUCTURES ON AFFINE SOLVMANIFOLDS
}

\author{
Alberto Medina And Mohamed Boucetta
}

\begin{abstract}
A $n$-dimensional Lie group $G$ equipped with a left invariant symplectic form $\omega^{+}$is called a symplectic Lie group. It is well known that $\omega^{+}$induces a left invariant affine structure on $G$. Relative to this affine structure we show that the left invariant Poisson tensor $\pi^{+}$corresponding to $\omega^{+}$is polynomial of degree at most 1 and any right invariant $k$-multivector field on $G$ is polynomial of degree at most $k$. If $G$ is unimodular, the symplectic form $\omega^{+}$is also polynomial and the volume form $\wedge^{n / 2} \omega^{+}$is parallel. We show also that any left invariant tensor field on a nilpotent symplectic Lie group is polynomial, in particular, any left invariant Poisson structure on a nilpotent symplectic Lie group is polynomial. Because many symplectic Lie groups admit uniform lattices, we get a large class of polynomial Poisson structures on compact affine solvmanifolds.
\end{abstract}

\section{Introduction and main results}

Recall that an affine manifold is a differential manifold $M$ together with a special atlas of coordinate charts such that all coordinate changes extend to affine automorphisms of $\mathbb{R}^{n}$. These distinguished charts are called affine charts. The data of a flat and torsion free connection $\nabla$ on a manifold $M$ is equivalent to the data of an affine structure.

A tensor field on an affine manifold $M$ is called polynomial if in affine coordinates its coefficients are polynomial functions. A Poisson structure on an affine manifold is called polynomial if the space of local polynomial functions is closed under the Poisson bracket. In an equivalent way this means that the associated Poisson bivector is polynomial. For some general results on polynomial tensor fields see $[\mathbf{6 , ~ 8 - 1 0 , 1 8}]$. Let us describe briefly the affine structure associated to a Lie group endowed with a left invariant symplectic form. This affine structure is the context on which we will state 
our main results on the polynomial nature of some tensor fields and some Poisson structures.

Let $G$ be a Lie group with Lie algebra $\mathfrak{g}=T_{e} G$, where $e$ stands for the unit of $G$. For any tensor $T$ on $\mathfrak{g}$, we denote by $T^{+}$and $T^{-}$respectively the left invariant tensor field and the right invariant tensor field on $G$ associated to $T$. If $\omega$ is a scalar nondegenerate 2-cocycle of $\mathfrak{g}$, the differential two-form $\omega^{+}$on $G$ is a left invariant symplectic form on $G$ and $\left(G, \omega^{+}\right)$is called a symplectic Lie group. Symplectic Lie groups were studied by several authors see, for instance, $[\mathbf{1}-\mathbf{5}, \mathbf{7}, \mathbf{1 2}, \mathbf{1 3}, \mathbf{1 5}, \mathbf{1 6}]$. A connected Lie group $G$ is symplectic if and only if its universal covering $\hat{G}$ admits an etale representation by affine transformations of $\mathfrak{g}^{*}$ with linear part the coadjoint representation of $\hat{G}$ and infinitesimal part a skew-symmetric 1-cocycle (see [14]). This means that we have a representation of the Lie algebra $\mathfrak{g}$ by affine endomorphisms of $\mathfrak{g}^{*}$ given by $u \mapsto\left(q(u), a d_{x}^{*}\right)$ with $q: \mathfrak{g} \longrightarrow \mathfrak{g}^{*}, u \mapsto q(u)$ invertible and skew-symmetric (see [14]). This implies that the formula

$$
\omega^{+}\left(\nabla_{u^{+}} v^{+}, w^{+}\right)=-\omega^{+}\left(v^{+},\left[u^{+}, w^{+}\right]\right),
$$

where $u, v, w \in \mathfrak{g}$, defines a left invariant flat and torsion-free connection $\nabla$. This affine structure will be called the affine structure associated to the symplectic Lie group $\left(G, \omega^{+}\right)$.

Let us state our main results.

Theorem 1.1. Let $\left(G, \omega^{+}\right)$be a connected symplectic Lie group of dimension $n$ endowed with the associated affine structure. Then the following assertions hold.

(1) In a neighborhood of any element of $G$, there exists an affine chart $\left(x_{1}, \ldots, x_{n}\right)$ such that, for any $i, j=1, \ldots, n$, the Poisson bracket of $x_{i}$ and $x_{j}$ associated to $\omega^{+}$is given by

$$
\left\{x_{i}, x_{j}\right\}=\sum_{k=1}^{n} C_{i j}^{k} x_{k}+\omega^{+}\left(X_{x_{i}}, X_{x_{j}}\right)(e),
$$

where $C_{i j}^{k}$ are the constants of structure of the Lie algebra of $G$ and $X_{x_{i}}$ is the Hamiltonian vector field associated to $x_{i}$.

(2) Any right invariant $k$-multivector field on $G$ is polynomial of degree at most $k$.

(3) If $G$ is unimodular then the symplectic form $\omega^{+}$is polynomial of degree at most $n-1$, the volume form $\wedge^{n / 2} \omega^{+}$is parallel and any right invariant differential form on $G$ is polynomial.

Theorem 1.2. Let $\left(G, \omega^{+}\right)$be a connected nilpotent symplectic Lie group endowed with the associated affine structure. Then any left invariant multivector field on $G$ is polynomial. In particular, any left invariant Poisson structure on $G$ is polynomial. 
There are some interesting implications of Theorems 1.1 and 1.2.

(1) Let $\left(G, \omega^{+}\right)$be a connected $n$-dimensional symplectic Lie group. If $G$ admits an uniform lattice, it is well known that $G$ is unimodular. On the other hand, according to a result of Medina-Lichnerowicz [13], the associated affine structure to $\left(G, \omega^{+}\right)$is geodesically complete if and only if $G$ is unimodular and, in this case $G$ is solvable.

Consequently if $\Gamma$ is an (uniform) lattice in $G$ then $M=\Gamma \backslash G$ is a compact solvmanifold which carries an affine structure and a symplectic form $\widetilde{\omega}$ such that:

(a) the Poisson bracket corresponding to $\widetilde{\omega}$ is polynomial of degree 1;

(b) the symplectic form $\widetilde{\omega}$ is polynomial of degree at most $n-1$ and the volume form $\wedge^{\frac{n}{2}} \widetilde{\omega}$ is parallel. Note that a compact affine manifold with a parallel volume form possesses interesting properties (see $[\mathbf{1 1}])$.

(2) Let $\left(G, \omega^{+}\right)$be a connected nilpotent symplectic Lie group. Then:

(a) any exact Lie-Poisson tensor on $G$ is polynomial;

(b) for any uniform lattice $\Gamma$ on $G$, any solution of the classical YangBaxter equation on $\mathfrak{g}$ gives arise to a left invariant Poisson tensor on $G$ which projects on a polynomial Poisson tensor on $M=\Gamma \backslash G$.

(3) Let $\left(G, \omega^{+}\right)$be a symplectic Lie group and let $r \in \mathfrak{g} \wedge \mathfrak{g}$ be the solution of the classical Yang-Baxter equation associated to $\omega$. According to Theorem 1.1, $r^{+}$is a polynomial Poisson structure of degree 1 and $r^{-}$is a polynomial Poisson structure of degree at most 2. Thus, we recover a result of Diatta-Medina (see [7]) which states that the LiePoisson bivector $r^{+}-r^{-}$is polynomial of degree 2 .

The paper is organized as follows. In Section 2, we give some properties of the affine structure associated to a symplectic Lie group. Proofs of Theorems 1.1 and 1.2 are developed in Section 3. Using Theorem 1.1 and results of Medina-Revoy on lattices in symplectic Lie groups [16], we exhibit in Section 4 and infinity of nonhomeomorphic compact affine solvmanifolds endowed with polynomial Poisson tensors.

\section{Some properties of the affine structure associated to a symplectic Lie group}

This section is a preparation of Section 3 in which we will prove Theorems 1.1 and 1.2 .

First, we will consider the affine structure given by (1.1) from a different point of view which will be useful through the paper.

Let $\pi^{+}$be a left invariant Poisson bivector on a Lie group $G$ and denote by $\pi_{\#}^{+}: T^{*} G \longrightarrow T G$ the associated homomorphism given by $\beta\left(\pi_{\#}^{+}(\alpha)\right)=$ 
$\pi^{+}(\alpha, \beta)$. Recall that the Koszul bracket associated to $\pi^{+}$is given by

$$
[\alpha, \beta]_{\pi^{+}}=\mathcal{L}_{\pi_{\#}^{+}(\alpha)} \beta-\mathcal{L}_{\pi_{\#}^{+}(\beta)} \alpha-d \pi^{+}(\alpha, \beta),
$$

where $\alpha$ and $\beta$ are differential one-forms on $G$ and $\mathcal{L}$ denotes the Lie derivative. This bracket endows $\Omega^{1}(G)$ with a structure of a Lie algebra and, for any $\alpha, \beta \in \Omega^{1}(G)$,

$$
\pi_{\#}^{+}\left([\alpha, \beta]_{\pi^{+}}\right)=\left[\pi_{\#}^{+}(\alpha), \pi_{\#}^{+}(\beta)\right] .
$$

An easy calculation gives that, for any vector field $X$ on $G$ and for any differential one-form $\alpha$,

$$
\left[\pi_{\#}^{+}(\alpha), X\right]=-\left(\mathcal{L}_{X} \pi^{+}\right)_{\#}(\alpha)-\pi_{\#}^{+}\left(\mathcal{L}_{X} \alpha\right) .
$$

We deduce easily from (2.1) that for any right invariant one-forms $\alpha^{-}$and $\beta^{-}$and for any right invariant vector field $X^{-}$,

$$
\left[\alpha^{-}, \beta^{-}\right]_{\pi^{+}}\left(X^{-}\right)=-\mathcal{L}_{X^{-}} \pi^{+}\left(\alpha^{-}, \beta^{-}\right)=0 .
$$

Thus, for any right invariant one-forms $\alpha^{-}$and $\beta^{-}$, the bracket $\left[\alpha^{-}, \beta^{-}\right]_{\pi^{+}}$ vanishes and hence

$$
\left[\pi_{\#}^{+}\left(\alpha^{-}\right), \pi_{\#}^{+}\left(\beta^{-}\right)\right]=0 .
$$

With this remark in mind, we consider a connected symplectic Lie group $\left(G, \omega^{+}\right)$and we denote by $\pi^{+}$the associated left invariant Poisson tensor. From (2.2), for any basis $\left(\alpha_{1}^{-}, \ldots, \alpha_{n}^{-}\right)$of right invariant one-forms on $G$, $\left(\pi_{\#}^{+}\left(\alpha_{1}^{-}\right), \ldots, \pi_{\#}^{+}\left(\alpha_{n}^{-}\right)\right)$is a commuting parallelism of vector fields on $G$. This defines a flat and torsion free linear connection $\widetilde{\nabla}$ by putting

$$
\widetilde{\nabla} \pi_{\#}^{+}\left(\alpha^{-}\right)=0
$$

for any right invariant one-form on $G$. Moreover, for any right invariant vector field $X^{-}$, we get from $(2.1)$

$$
\left[\pi_{\#}^{+}\left(\alpha^{-}\right), X^{-}\right]=\widetilde{\nabla}_{\pi_{\#}^{+}\left(\alpha^{-}\right)} X^{-}=-\pi_{\#}^{+}\left(\mathcal{L}_{X^{-}} \alpha^{-}\right),
$$

and since $\mathcal{L}_{X^{-}} \alpha^{-}$is a right invariant one-form, we get

$$
\widetilde{\nabla} \widetilde{\nabla}_{\pi_{\#}^{+}\left(\alpha^{-}\right)} X^{-}=0
$$

Then $X^{-}$is an affine infinitesimal transformation and hence $\widetilde{\nabla}$ is a left invariant linear connection.

Now, let us compare the linear connection $\nabla$ defined by $(1.1)$ and $\widetilde{\nabla}$. More precisely, we will show that

$$
\widetilde{\nabla}=\nabla .
$$

The symplectic form $\omega^{+}$gives arise to an isomorphism $\omega^{b}: T G \longrightarrow T^{*} G$, $u \mapsto \omega^{+}(u,$.$) . This isomorphism and its inverse \left(\omega^{b}\right)^{-1}: T^{*} G \longrightarrow T G$ define 
an isomorphism between the space of tensor field of type $(p, q)$ and the space of tensor field of type $(q, p)$. For any tensor field $T$, we denote by $T^{\omega}$ its image under this isomorphism. For instance, for any vector field $X$ and any one-form $\alpha, X^{\omega}$ is the one-form $i_{X} \omega^{+}$and $\alpha^{\omega}=-\pi_{\#}^{+}(\alpha)$. It is obvious that $\left(T^{\omega}\right)^{\omega}=T$.

Proposition 2.1. Let $\left(G, \omega^{+}\right)$be a symplectic Lie group. Then:

(1) For any left invariant vector field $X^{+}$and for any tensor field $T$, we have

$$
\nabla_{X^{+}} T=\left(\mathcal{L}_{X^{+}} T^{\omega}\right)^{\omega},
$$

where $\mathcal{L}_{X^{+}}$is the Lie derivative in the direction of $X^{+}$.

(2) A tensor field $T$ is parallel with respect to $\nabla$ if and only if $T^{\omega}$ is right invariant.

Proof. Note that the second assertion is an immediate consequence of the first one. Let us establish the first assertion. Since, for any tensor fields $T_{1}$ and $T_{2},\left(T_{1} \otimes T_{2}\right)^{\omega}=T_{1}^{\omega} \otimes T_{2}^{\omega}$ and since both $\mathcal{L}_{X^{+}}$and $\nabla_{X^{+}}$are derivative, it suffices to establish the relation for left invariant vector fields and left invariant differential one-forms. Let $Y^{+}$and $Z^{+}$be left invariant vector fields. We have

$$
\begin{aligned}
\omega^{+}\left(\left(\mathcal{L}_{X^{+}} i_{Y^{+}} \omega^{+}\right)^{\omega}, Z^{+}\right) & =\mathcal{L}_{X^{+}} i_{Y^{+}} \omega^{+}\left(Z^{+}\right) \\
& =X^{+} \cdot \omega^{+}\left(Y^{+}, Z^{+}\right)-\omega^{+}\left(Y^{+},\left[X^{+}, Z^{+}\right]\right) \\
& =-\omega^{+}\left(Y^{+},\left[X^{+}, Z^{+}\right]\right)=\omega^{+}\left(\nabla_{X^{+}} Y^{+}, Z^{+}\right),
\end{aligned}
$$

and the formula follows. One can easily deduce the formula for a left invariant one-form.

An immediate consequence of this proposition is that, $\nabla \pi_{\#}^{+}\left(\alpha^{-}\right)=0$ for any right invariant one-form $\alpha^{-}$and hence $\widetilde{\nabla}=\nabla$.

Now, given a connected symplectic Lie group $\left(G, \omega^{+}\right)$, let us construct an affine atlas corresponding $\nabla$.

Since $\omega^{+}$is left invariant, for any $u \in \mathfrak{g}$, the vector field $u^{-}$is symplectic, i.e.,

$$
\mathcal{L}_{u^{-}} \omega^{+}=d i_{u^{-}} \omega^{+}=0 \text {. }
$$

Thus, for any basis $\left(u_{1}, \ldots, u_{n}\right)$ of $\mathfrak{g}$, there exists in a neighborhood of any element of $G$ a local coordinates $\left(x_{1}, \ldots, x_{n}\right)$ such that

$$
i_{u_{i}^{-} \omega^{+}}=d x_{i}, \quad i=1, \ldots, n .
$$

We get from Proposition 2.1 that $\nabla d x_{i}=0$ and we deduce that $\left(x_{1}, \ldots, x_{n}\right)$ are affine coordinates. 
Now we will express $\omega^{+}$and $\pi^{+}$in the affine coordinates constructed above. Fix a basis $\left(u_{1}, \ldots, u_{n}\right)$ of $\mathfrak{g}$, denote by $\left(\alpha_{1}, \ldots, \alpha_{n}\right)$ its dual basis and consider the affine coordinates $\left(x_{1}, \ldots, x_{n}\right)$ given by $(2.3)$. In this coordinates we have

$$
\begin{aligned}
& \omega^{+}=\sum_{i<j} \pi^{+}\left(\alpha_{i}^{-}, \alpha_{j}^{-}\right) d x_{i} \wedge d x_{j}, \\
& \pi^{+}=\sum_{i<j} \omega^{+}\left(u_{i}^{-}, u_{j}^{-}\right) \frac{\partial}{\partial x_{i}} \wedge \frac{\partial}{\partial x_{j}}, \\
& u^{-}=\sum_{j=1}^{n} \omega^{+}\left(u_{j}^{-}, u^{-}\right) \frac{\partial}{\partial x_{j}}, \quad u \in \mathfrak{g}, \\
& \alpha^{-}=\sum_{j=1}^{n} \pi^{+}\left(\alpha_{j}^{-}, \alpha^{-}\right) d x_{j}, \quad \alpha \in \mathfrak{g}^{*} .
\end{aligned}
$$

The following proposition will play a crucial role in the proof of Theorem 1.1.

Proposition 2.2. Let $\left(G, \omega^{+}\right)$be a connected symplectic Lie group endowed with the associated affine structure. Then, for any $u, v \in \mathfrak{g}$ such that $[u, v] \neq$ $0, \omega^{+}\left(u^{-}, v^{-}\right)$is a polynomial function of degree 1 .

Proof. The right invariant vector fields $u^{-}, v^{-}$are symplectic and hence $\left[u^{-}, v^{-}\right]$is Hamiltonian and we have

$$
i_{\left[u^{-}, v^{-}\right]} \omega^{+}=d \omega^{+}\left(u^{-}, v^{-}\right) .
$$

By using Proposition 2.1, we get $\nabla d \omega^{+}\left(u^{-}, v^{-}\right)=0$ and the result follows.

Note that if $A$ is the matrix $\left(\omega^{+}\left(u_{i}^{-}, u_{j}^{-}\right)\right)_{1 \leq i, j \leq n}$, we have

$$
A^{-1}=\left(\pi^{+}\left(\alpha_{i}^{-}, \alpha_{j}^{-}\right)\right)_{1 \leq i, j \leq n} .
$$

Consider now the volume form $\Omega^{+}=\wedge^{\frac{n}{2}} \omega^{+}$. We have, from (2.6),

$$
\Omega^{+}\left(u_{1}^{-}, \ldots, u_{n}^{-}\right)=\operatorname{det}(A) \Omega^{+}\left(\frac{\partial}{\partial x_{1}}, \ldots, \frac{\partial}{\partial x_{n}}\right),
$$

and

$$
\operatorname{det}(A)=\frac{1}{\left(\left(\frac{n}{2}\right) !\right)^{2}} \Omega^{+}\left(u_{1}^{-}, \ldots, u_{n}^{-}\right)^{2} .
$$

Proposition 2.3. Let $\left(G, \omega^{+}\right)$be a connected unimodular symplectic Lie group endowed with the associated affine structure and let $\pi^{+}$be the left invariant Poisson structure corresponding to $\omega^{+}$. Then $\pi^{+}\left(\alpha^{-}, \beta^{-}\right)$is a polynomial function of degree at most $n-1$, for any differential forms $\alpha^{-}, \beta^{-}$. 
Proof. Since $G$ is unimodular, $\Omega^{+}$is a right invariant and then $\Omega^{+}$ $\left(u_{1}^{-}, \ldots, u_{n}^{-}\right)$is constant. Hence, from $(2.10), \operatorname{det}(A)$ is a constant. On the other hand, from Proposition 2.2, the coefficients of $A$ are polynomial functions of degree 1 , consequently the coefficients of the inverse $A^{-1}$ are polynomial of degree at most $n-1$ and the proposition follows from (2.8).

The following Lemma will be useful in the proof of Theorem 1.2.

Lemma 2.1. A function $f$ on an unimodular symplectic Lie group $\left(G, \omega^{+}\right)$ is polynomial if and only if $u^{-}(f)$ is polynomial for any $u \in \mathfrak{g}$.

Proof. We have from (2.6)

$$
u^{-}(f)=\sum_{j=1}^{n} \omega^{+}\left(u_{j}^{-}, u^{-}\right) \frac{\partial f}{\partial x_{j}} .
$$

So if $f$ is polynomial, $u^{-}(f)$ is polynomial according to Proposition 2.2. For the converse, we deduce from 2.11 that

$$
\left(\begin{array}{c}
\frac{\partial f}{\partial x_{1}} \\
\vdots \\
\frac{\partial f}{\partial x_{n}}
\end{array}\right)=A^{-1}\left(\begin{array}{c}
u_{1}^{-}(f) \\
\vdots \\
u_{n}^{-}(f)
\end{array}\right) \text {. }
$$

But, we have see in the proof of Proposition 2.3 that if $G$ is unimodular the coefficients of $A^{-1}$ are polynomial and the Lemma follows.

\section{Proof of Theorems 1.1 and 1.2}

\subsection{Proof of Theorem 1.1.}

(1) Fix a basis $\left(u_{1}, \ldots, u_{n}\right)$ of $\mathfrak{g}$, denote by $\left(\alpha_{1}, \ldots, \alpha_{n}\right)$ its dual basis and consider the affine coordinates $\left(x_{1}, \ldots, x_{n}\right)$ given by $(2.3)$. For any $u_{i}, u_{j}$, put $\left[u_{i}, u_{j}\right]=\sum_{k=1}^{n} C_{i j}^{k} u_{k}$.

From $(2.5)$, we have $\left\{x_{i}, x_{j}\right\}=\omega^{+}\left(u_{i}^{-}, u_{j}^{-}\right)$. Hence, for any $1 \leq$ $i, j, k \leq n$,

$$
\begin{aligned}
\frac{\partial}{\partial x_{k}} \cdot\left\{x_{i}, x_{j}\right\} & =d \omega^{+}\left(u_{i}^{-}, u_{j}^{-}\right)\left(\pi_{\#}^{+}\left(\alpha_{k}^{-}\right)\right) \\
& =i_{\left[u_{i}^{-}, u_{j}^{-}\right]} \omega^{+}\left(\pi_{\#}^{+}\left(\alpha_{k}^{-}\right)\right) \\
& =\alpha_{k}^{-}\left(\left[u_{i}^{-}, u_{j}^{-}\right]\right)=C_{i j}^{k},
\end{aligned}
$$

thus $d\left\{x_{i}, x_{j}\right\}=\sum_{k=1}^{n} C_{i j}^{k} d x_{k}$ and the desired relation follows.

(2) From (2.6) and Proposition 2.2 we deduce that any right invariant vector field on $G$ is polynomial of degree at most 1 and hence any right invariant $k$-multivector field must be polynomial of degree at most $k$. 
(3) This is an immediate consequence of Proposition 2.3, (2.4), (2.8) and (2.9).

3.2. Proof of Theorem 1.2. To prove the theorem, it suffices to show that if $G$ is nilpotent then any left invariant vector field is polynomial. Fix a basis $\left(u_{1}, \ldots, u_{n}\right)$ of $\mathfrak{g}$ and consider the affine coordinates $\left(x_{1}, \ldots, x_{n}\right)$ given by $(2.3)$. Let $u^{+}$be a left invariant vector field on $G$. We have

$$
u^{+}=\sum_{i=1}^{n} \omega^{+}\left(u_{i}^{-}, u^{+}\right) \frac{\partial}{\partial x_{i}} .
$$

Since $\mathcal{L}_{u_{j}^{-}} \omega^{+}=0$ and $\left[u_{j}^{-}, u^{+}\right]=0$, we have, for any $1 \leq i, j \leq n$,

$$
u_{j}^{-} \cdot \omega^{+}\left(u_{i}^{-}, u^{+}\right)=\omega^{+}\left(\left[u_{j}^{-}, u_{i}^{-}\right], u^{+}\right)
$$

and by induction, we get

$$
u_{j_{1}}^{-} \circ \ldots \circ u_{j_{r}}^{-} \cdot \omega^{+}\left(u_{i}^{-}, u^{+}\right)=\omega^{+}\left(\left[u_{j_{1}}^{-},\left[\ldots,\left[u_{j_{r}}^{-}, u_{i}^{-}\right]\right], u^{+}\right),\right.
$$

for any $1 \leq j_{1}, \ldots, j_{r} \leq n$. Since $G$ is nilpotent, we get for $r$ large

$$
u_{j_{1}}^{-} \circ \ldots \circ u_{j_{r}}^{-} \cdot \omega^{+}\left(u_{i}^{-}, u^{+}\right)=0
$$

and we deduce from Lemma 2.1 that $\omega^{+}\left(u_{i}^{-}, u^{+}\right)$is a polynomial function and the theorem is proved.

\section{Examples}

In this section, we give a large class of four-dimensional solvmanifolds which admit polynomial symplectic forms and polynomial Poisson structures. The construction is based on Theorems 1.1 and 1.2 and on the results of MedinaRevoy on Lattices in four-dimensional symplectic Lie groups (see [16]). Indeed, in [15] Medina and Revoy showed that there are four non-Abelian real unimodular Lie algebras of dimension four endowed with a scalar nondegenerate 2-cocycle. Moreover, the connected and simply connected Lie group of any of such Lie algebras has an infinity of nonisomorphic lattices. For any Lie algebra in the list of Medina-Revoy, we consider the corresponding connected and simply connected Lie group endowed with a left invariant symplectic form, we give a global affine chart and we express the symplectic form and the Poisson bivector in this chart. Finally, we give a description of lattices in this group.

Example 4.1. (1) We consider the Lie group $G_{1}=\mathbb{R}^{4}$ with the product

$$
(x, y, z, t)\left(x^{\prime}, z^{\prime}, y^{\prime}, t^{\prime}\right)=\left(x+x^{\prime}, y+y^{\prime}+t x^{\prime}, z+z^{\prime}+t y^{\prime}+\frac{t^{2}}{2} x^{\prime}, t+t^{\prime}\right) .
$$


We denote by $\mathfrak{g}_{1}$ its Lie algebra and by $\left(e_{1}, e_{2}, e_{3}, e_{4}\right)$ the canonical basis of $\mathfrak{g}_{1}$. We have

$$
\begin{aligned}
& e_{1}^{+}=\partial_{x}+t \partial_{y}+\frac{t^{2}}{2} \partial_{z}, \quad e_{2}^{+}=\partial_{y}+t \partial_{z}, \quad e_{3}^{+}=\partial_{z}, \quad e_{4}^{+}=\partial_{t} \\
& e_{1}^{-}=\partial_{x}, \quad e_{2}^{-}=\partial_{y}, \quad e_{3}^{-}=\partial_{z}, \quad e_{4}^{-}=x \partial_{y}+y \partial_{z}+\partial_{t}
\end{aligned}
$$

The nonzero brackets of $\mathfrak{g}_{1}$ are the following:

$$
\left[e_{4}, e_{1}\right]=e_{2} \quad \text { and } \quad\left[e_{4}, e_{2}\right]=e_{3} .
$$

We consider the scalar nondegenerate 2-cocycle on $\mathfrak{g}_{1}$ given by $\omega=$ $e_{4}^{*} \wedge e_{3}^{*}+e_{1}^{*} \wedge e_{2}^{*}$. A direct computation gives that the corresponding symplectic two-form on $G_{1}$ is given by

$$
\omega^{+}=d x \wedge d y-\frac{1}{2} t^{2} d x \wedge d t+t d y \wedge d t-d z \wedge d t
$$

and

$$
\begin{aligned}
& i_{e_{1}^{-} \omega^{+}}=d\left(y-\frac{1}{6} t^{3}\right), \quad i_{e_{2}^{-} \omega^{+}}=d\left(-x+\frac{1}{2} t^{2}\right) \\
& i_{e_{3}^{-} \omega^{+}}=-d t, \quad i_{e_{4}^{-} \omega^{+}}=d\left(-\frac{1}{2} x^{2}+\frac{1}{2} x t^{2}-y t+z\right) .
\end{aligned}
$$

We put

$$
X=y-\frac{1}{6} t^{3}, Y=-x+\frac{1}{2} t^{2}, Z=-t \quad \text { and } \quad T=-\frac{1}{2} x^{2}+\frac{1}{2} x t^{2}-y t+z,
$$

and we get an affine chart $(X, Y, Z, T)$.

Here the matrix $A=\left(\omega^{+}\left(e_{i}^{-}, e_{j}^{-}\right)\right)_{1 \leq i, j \leq 4}$ is given by

$$
A=\left(\begin{array}{rrrr}
0 & 1 & 0 & -Y \\
-1 & 0 & 0 & -Z \\
0 & 0 & 0 & -1 \\
Y & Z & 1 & 0
\end{array}\right)
$$

and hence, by using (2.5),

$$
\pi^{+}=\partial_{X} \wedge \partial_{Y}-Y \partial_{X} \wedge \partial_{T}-Z \partial_{Y} \wedge \partial_{T}-\partial_{Z} \wedge \partial_{T}
$$

The inverse of $A$ is given by

$$
A^{-1}=\left(\begin{array}{rrrr}
0 & -1 & Z & 0 \\
1 & 0 & -Y & 0 \\
-Z & Y & 0 & 1 \\
0 & 0 & -1 & 0
\end{array}\right)
$$


and hence

$$
\omega^{+}=-d X \wedge d Y+Z d X \wedge d Z-Y d Y \wedge d Z+d Z \wedge d T .
$$

It is clear that $\Gamma=\{(m, n, k, 2 r) / m, n, k, r \in \mathbb{Z}\}$ is an uniform lattice in $G_{1}$. Actually, $G_{1}$ admits an infinity nonisomorphic lattices.

(2) We consider the Lie group $G_{2}=\mathbb{R}^{4}$ with the product

$$
(x, y, z, t)\left(x^{\prime}, y^{\prime}, z^{\prime}, t^{\prime}\right)=\left(x+x^{\prime}, y+e^{x} y^{\prime}, z+e^{-x} z^{\prime}, t+t^{\prime}\right) .
$$

We denote by $\mathfrak{g}_{2}$ its Lie algebra and by $\left(e_{1}, e_{2}, e_{3}, e_{4}\right)$ the canonical basis of $\mathfrak{g}_{2}$. We have

$$
\begin{aligned}
& e_{1}^{+}=\partial_{x}, \quad e_{2}^{+}=e^{x} \partial_{y}, \quad e_{3}^{+}=e^{-x} \partial_{z}, \quad e_{4}^{+}=\partial_{t}, \\
& e_{1}^{-}=\partial_{x}+y \partial_{y}-z \partial_{z}, \quad e_{2}^{-}=\partial_{y}, \quad e_{3}^{-}=\partial_{z}, \quad e_{4}^{-}=\partial_{t} .
\end{aligned}
$$

The nonzero brackets of $\mathfrak{g}_{2}$ are the following:

$$
\left[e_{1}, e_{2}\right]=e_{2} \quad \text { and } \quad\left[e_{1}, e_{3}\right]=-e_{3} .
$$

We consider the scalar nondegenerate 2-cocycle on $\mathfrak{g}_{2}$ given by $\omega=$ $e_{1}^{*} \wedge e_{4}^{*}+e_{2}^{*} \wedge e_{3}^{*}$. A direct computation gives that the corresponding symplectic two-form on $G_{2}$ is given by

$$
\omega^{+}=d x \wedge d t+d y \wedge d z
$$

and

$$
i_{e_{1}^{-} \omega^{+}}=d(t+y z), i_{e_{2}^{-} \omega^{+}}=d z, i_{e_{3}^{-} \omega^{+}}=-d y \quad \text { and } \quad i_{e_{4}^{-}} \omega^{+}=-d x \text {. }
$$

We put $(X, Y, Z, T)=(t+y z, z,-y,-x)$ and we get an affine chart.

Here the matrix $A=\left(\omega^{+}\left(e_{i}^{-}, e_{j}^{-}\right)\right)_{1 \leq i, j \leq 4}$ is given by

$$
A=\left(\begin{array}{cccc}
0 & -Y & -Z & 1 \\
Y & 0 & 1 & 0 \\
Z & -1 & 0 & 0 \\
-1 & 0 & 0 & 0
\end{array}\right)
$$

and hence, by using (2.5),

$$
\pi^{+}=-Y \partial_{X} \wedge \partial_{Y}-Z \partial_{X} \wedge \partial_{Z}+\partial_{X} \wedge \partial_{T}+\partial_{Y} \wedge \partial_{Z}
$$

The inverse of $A$ is given by

$$
A^{-1}=\left(\begin{array}{cccc}
0 & 0 & 0 & -1 \\
0 & 0 & -1 & -Z \\
0 & 1 & 0 & Y \\
1 & Z & -Y & 0
\end{array}\right)
$$


and hence

$$
\omega^{+}=-d X \wedge d T-d Y \wedge d Z-Z d Y \wedge d T+Y d Z \wedge d T .
$$

The Lie group $G_{2}$ is a direct product of $G_{2}^{\prime}$ with the Abelian group $\mathbb{R}$ where the multiplication in $G_{2}^{\prime}$ is given by

$$
(x, y, z)\left(x^{\prime}, y^{\prime}, z^{\prime}\right)=\left(x+x^{\prime}, y+e^{x} y^{\prime}, z+e^{-x} z^{\prime}\right) .
$$

Hence, if $\Gamma_{1}$ is a lattice in $G_{2}^{\prime}$ then $\Gamma=\Gamma_{1} \times \mathbb{Z}$ is a lattice in $G_{2}$. But, according to $[\mathbf{1 3}]$, a lattice $\Gamma_{1}$ in $G_{2}^{\prime}$ is a semi-direct product of $\mathbb{Z}$ by $\mathbb{Z}^{2}$ by an action $\phi$ of $\mathbb{Z}$ on $\mathbb{Z}^{2}$ with $\phi(1)$ acts as $A=\left(\begin{array}{cc}0 & -1 \\ 1 & n\end{array}\right)(n \geq 3)$ in a suitable basis of $\Gamma_{1} \cap N$, where $N$ is nilradical of $G_{2}^{\prime}$.

(3) We consider the Lie group $G_{3}=\mathbb{R}^{4}$ with the product

$$
\begin{aligned}
(x, y, z, t)\left(x^{\prime}, y^{\prime}, z^{\prime}, t^{\prime}\right)= & \left(x+x^{\prime}, y+y^{\prime} \cos (x)-z^{\prime} \sin (x),\right. \\
& \left.z+y^{\prime} \sin (x)+z^{\prime} \cos (x), t+t^{\prime}\right) .
\end{aligned}
$$

We denote by $\mathfrak{g}_{3}$ its Lie algebra and by $\left(e_{1}, e_{2}, e_{3}, e_{4}\right)$ the canonical basis of $\mathfrak{g}_{3}$. We have

$$
\begin{aligned}
& e_{1}^{+}=\partial_{x}, \quad e_{2}^{+}=\cos x \partial_{y}+\sin x \partial_{z}, \quad e_{3}^{+}=-\sin x \partial_{y}+\cos x \partial_{z}, \quad e_{4}^{+}=\partial_{t}, \\
& e_{1}^{-}=\partial_{x}-z \partial_{y}+y \partial_{z}, \quad e_{2}^{-}=\partial_{y}, \quad e_{3}^{-}=\partial_{z}, \quad e_{4}^{-}=\partial_{t},
\end{aligned}
$$

The nonzero brackets of $\mathfrak{g}_{3}$ are the following:

$$
\left[e_{1}, e_{2}\right]=e_{3} \quad \text { and } \quad\left[e_{1}, e_{3}\right]=-e_{2} .
$$

We consider the scalar nondegenerate 2-cocycle on $\mathfrak{g}_{3}$ given by $\omega=$ $e_{1}^{*} \wedge e_{4}^{*}+e_{2}^{*} \wedge e_{3}^{*}$. A direct computation gives that the corresponding symplectic two-form on $G_{2}$ is given by

$$
\omega^{+}=d x \wedge d t+d y \wedge d z
$$

and

$$
i_{e_{1}^{-} \omega^{+}}=d(t-y z), i_{e_{2}^{-} \omega^{+}}=d z, i_{e_{3}^{-} \omega^{+}}=-d y \quad \text { and } \quad i_{e_{4}^{-}} \omega^{+}=-d x \text {. }
$$

We put $(X, Y, Z, T)=(t-y z, z,-y,-x)$ and we get an affine chart.

The matrix $A=\left(\omega^{+}\left(e_{i}^{-}, e_{j}^{-}\right)\right)_{1 \leq i, j \leq 4}$ is given by

$$
A=\left(\begin{array}{cccc}
0 & Z & -Y & 1 \\
-Z & 0 & 1 & 0 \\
Y & -1 & 0 & 0 \\
-1 & 0 & 0 & 0
\end{array}\right)
$$


and hence, by using (2.5),

$$
\pi^{+}=Z \partial_{X} \wedge \partial_{Y}-Y \partial_{X} \wedge \partial_{Z}+\partial_{X} \wedge \partial_{T}+\partial_{Y} \wedge \partial_{Z}
$$

The inverse of $A$ is given by

$$
A^{-1}=\left(\begin{array}{cccc}
0 & 0 & 0 & -1 \\
0 & 0 & -1 & -Y \\
0 & 1 & 0 & -Z \\
1 & Y & Z & 0
\end{array}\right)
$$

and hence

$$
\omega^{+}=-d X \wedge d T-d Y \wedge d Z-Y d Y \wedge d T-Z d Z \wedge d T .
$$

The Lie group $G_{3}$ is a direct product of $G_{3}^{\prime}$ with the Abelian group $\mathbb{R}$ where the multiplication on $G_{3}^{\prime}$ is given by

$$
\begin{aligned}
(x, y, z)\left(x^{\prime}, y^{\prime}, z^{\prime}\right)= & \left(x+x^{\prime}, y+y^{\prime} \cos (x)-z^{\prime} \sin (x),\right. \\
& \left.z+y^{\prime} \sin (x)+z^{\prime} \cos (x)\right) .
\end{aligned}
$$

Hence, if $\Gamma_{1}$ is a lattice in $G_{3}^{\prime}$ then $\Gamma=\Gamma_{1} \times \mathbb{Z}$ is a lattice in $G_{3}$. Now, the Lie group $G_{3}^{\prime}$, which is a semi-direct product of $\mathbb{R}$ with $\mathbb{R}^{2}$, is isomorphic to the universal covering of the positive motions of the Euclidian plan. The element $1 \in \mathbb{R}$ and the standard lattice $\mathbb{Z}^{2}$ in $\mathbb{R}^{2}$ generates a lattice $\Gamma_{1}$ isomorphic to $\mathbb{Z}^{3}$. Moreover, if we consider the subgroup of $G_{3}^{\prime}$ generated by $\mathbb{Z}^{2}$ and the element $\frac{1}{2} \in \mathbb{R}$ then we get a lattice in $G_{3}^{\prime}$ containing $\Gamma_{1}$ as a subgroup of index 2 , which is not nilpotent.

(4) We consider $G_{4}=\mathbb{R}^{3} \times \mathbb{R}^{*+}$ the direct product of the threedimensional Heisenberg Lie group with the one-dimensional Lie group. The product is given by

$$
(x, y, z, t)\left(x^{\prime}, y^{\prime}, z^{\prime}, t^{\prime}\right)=\left(x+x^{\prime}, y+y^{\prime}, z+z^{\prime}+x y^{\prime}, t t^{\prime}\right) .
$$

We denote by $\mathfrak{g}_{4}$ its Lie algebra and by $\left(e_{1}, e_{2}, e_{3}, e_{4}\right)$ the canonical basis of $\mathfrak{g}_{4}$. We have

$$
\begin{aligned}
& e_{1}^{+}=\partial_{x}, \quad e_{2}^{+}=\partial_{y}+x \partial_{z}, \quad e_{3}^{+}=\partial_{z}, \quad e_{4}^{+}=t \partial_{t}, \\
& e_{1}^{-}=\partial_{x}+y \partial_{z}, \quad e_{2}^{-}=\partial_{y}, \quad e_{3}^{-}=\partial_{z}, \quad e_{4}^{-}=t \partial_{t} .
\end{aligned}
$$

The nonzero brackets of $\mathfrak{g}_{4}$ are the following:

$$
\left[e_{1}, e_{2}\right]=e_{3} \text {. }
$$

We consider the scalar nondegenerate 2-cocycle on $\mathfrak{g}_{4}$ given by $\omega=$ $e_{1}^{*} \wedge e_{4}^{*}+e_{2}^{*} \wedge e_{3}^{*}$. A direct computation gives that the corresponding symplectic two-form on $G_{4}$ is given by

$$
\omega^{+}=\frac{1}{t} d x \wedge d t+d y \wedge d z,
$$


and

$$
i_{e_{1}^{-} \omega^{+}}=d\left(\ln t-\frac{1}{2} y^{2}\right), i_{e_{2}^{-} \omega^{+}}=d z, i_{e_{3}^{-} \omega^{+}}=-d y, i_{e_{4}^{-}} \omega^{+}=-d x .
$$

We put $(X, Y, Z, T)=\left(\ln t-\frac{1}{2} y^{2}, z,-y,-x\right)$ and we get an affine chart.

The matrix $A=\left(\omega^{+}\left(e_{i}^{-}, e_{j}^{-}\right)\right)_{1 \leq i, j \leq 4}$ is given by

$$
A=\left(\begin{array}{cccc}
0 & Z & 0 & 1 \\
-Z & 0 & 1 & 0 \\
0 & -1 & 0 & 0 \\
-1 & 0 & 0 & 0
\end{array}\right)
$$

and hence, by using (2.5)

$$
\pi^{+}=Z \partial_{X} \wedge \partial_{Y}+\partial_{X} \wedge \partial_{T}+\partial_{Y} \wedge \partial_{Z}
$$

The inverse of $A$ is given by

$$
A^{-1}=\left(\begin{array}{cccc}
0 & 0 & 0 & -1 \\
0 & 0 & -1 & 0 \\
0 & 1 & 0 & -Z \\
1 & 0 & Z & 0
\end{array}\right)
$$

and hence

$$
\omega^{+}=-d X \wedge d T-d Y \wedge d Z-Z d Z \wedge d T .
$$

The group $G_{4}$ is a product of the Heisenberg group $N_{3}$ with the Abelian group $\mathbb{R}$. Note that $N_{3}$ is isomorphic to the group of all unipotent real upper triangular $3 \times 3$ matrix. The subset of $N_{3}$

$$
\Gamma_{p, q, r}=\left\{\left(\begin{array}{ccc}
1 & \frac{m}{p} & \frac{k}{p q r} \\
0 & 1 & \frac{n}{q} \\
0 & 0 & 1
\end{array}\right) ; m, n, k \in \mathbb{Z}\right\},
$$

where $p, q, r$ are fixed integers numbers with $p q r \neq 0$, is a lattice in $N_{3}$. It is commensurable with $\Gamma_{1,1,1}$. In fact, $\Gamma_{p, q, r}$ contains $\Gamma_{1,1,1}$ as a subgroup of index $p^{2} q^{2} r$ (see [17]).

To get examples in dimension greater or equal to 6 , one cane use the results given in $[\mathbf{1}]$ or $[\mathbf{1 2}]$. Note that Theorem 1.1 asserts in part 3 that $\Omega^{+}$ is of degree at most $n-1$. In all examples above the degree of $\omega^{+}$is 1 and it will be interesting to find examples in the four-dimensional case where the degree of $\omega^{+}$is 3 . 


\section{Acknowledgment}

This work was finalized during the stay of the first author at the university Montpelier II. The authors wish to thank the referee for his relevant remarks.

\section{References}

[1] I. Bajo, S. Benayadi and A. Medina, Symplectic structures on quadratic Lie algebras, J. Algebra 316 (1) (2007), 174-188.

[2] C. Benson and C. Gordon, Kähler and symplectic structures on nilmanifolds, Topology 27(4) (1988), 513-518.

[3] B.Y. Chu, Symplectic homogeneous spaces, Trans. Am. Math. Soc. 197 (1974), 145-159.

[4] J.M. Dardié and A. Medina, Groupes de Lie kahlriens et double extension, J. Algebra 185(3) (1996), 774-795.

[5] J.M. Dardié and A. Medina, Double extension symplectique d'un groupe de Lie symplectique, Adv. Math. 117(2) (1996), 208-227.

[6] K. Dekimpe, Polynomial structures and the uniqueness of affinely flat infra-nilmanifolds, Math. Z. 224(3) (1997), 457-481.

[7] A. Diatta and A. Medina, Classical Yang-Baxter equation and left invariant affine geometry on Lie groups, Manuscripta Math. 114(4) (2004), 477-486.

[8] D. Fried, Polynomials on affine manifolds, Trans. Amer. Math. Soc. 274(2) (1982), 709-719.

[9] M. Goldman, William and W. Hirsch, Morris, Polynomial forms on affine manifolds, Pac. J. Math. 101(1) (1982), 115-121.

[10] M. Goldman, William, On the polynomial cohomology of affine manifolds, Invent. Math. 65(3) (1981/82), 453-457.

[11] L. Huei Shyong, Note on affine manifold with parallel volume element, Geom. Dedicata 9(2) (1980), 195-198.

[12] Y. Khakimdjanov, M. Goze and A. Medina, Symplectic or contact structures on Lie groups, Differential Geom. Appl. 21(1) (2004), 41-54.

[13] A. Lichnerowicz and A. Medina, On Lie groups with left-invariant symplectic or Kählerian structures, Lett. Math. Phys. 16 (1988), 225-235.

[14] A. Medina, Structures de Poisson affines, symplectic geometry and mathematical physics, Progress In Mathematics, Birkhäuser, 1991.

[15] A. Medina and P. Revoy, Groupes de Lie à structure symplectique invariante, symplectic geometry, groupoids and integrable systems, in 'Séminaire Sud Rhodanien', M.S.R.I., New York/Berlin, Springer-Verlag, 1991, 247-266.

[16] A. Medina and P. Revoy, Lattices in Symplectic Lie Groups, J. Lie Theory, 17 (2007), 27-39.

[17] A.L Onishchik and E.B. Vinberg (Eds), Lie Groups and Lie Algebras II, Encyclopaedia of Mathematical Sciences, Vol 21, Springer-Verlag, Berlin, 2000.

[18] A. Tsemo, Automorphismes polynomiaux des variétés affines, C. R. Acad. Sci. Paris Sér. I Math. 329(11) (1999), 997-1002. 
UNIVERSité MONTPELLIER 2,

Case Courrier 051, UMR CNRS 5149,

Place Eugne Bataillon, 34095

MONTPELliER CEDEX, France

E-mail address: medina@math.univ-montp2.fr

Faculté des Sciences et Techniques,

BP 549, Marrakech, MorocCO

E-mail address: boucetta@fstg-marrakech.ac.ma

Received 06/25/2008, accepted 12/01/2010 
